\title{
APPLICATION POTENTIAL AND PLASTICITY OF HUMAN STEM CELLS
}

\author{
Mikołaj Lorenz ${ }^{1}$ Paul Mozdziak², Bartosz Kempisty ${ }^{1,3,4}$, Marta Dyszkiewicz-Konwińska ${ }^{1,5}$
}

\begin{abstract}
Significant advances have been achieved in the study of stem cells over recent years. Stem cell isolation, their plasticity, differentiation and pre-clinical and clinical applications have undergone a significant study. The objective of this paper is to review the advances in stem cell isolation methods. There are many types of stem cells in the article. Isolation and subsequent differentiation of among others: Human adipose-derived stem cells, cancer stem cells, neural stem cells and mesenchymal stem cells. The subject of Endometrial mesenchymal stromal cells, whose isolation methods are relatively new, was also raised. Attention was paid to the development of preclinical studies using Dental Pulp Stem Cells in various diseases such as Parkinson's disease or Alzheimer's disease. Progress in research on the use of stem cells in the treatment of heart attacks, burns, bone injuries and the use of neural stem cells in animal models as an attempt to treat multiple sclerosis has been described.
\end{abstract}

Running title: Potential and plasticity of stem cells

Keywords: origin of stem cells, isolation of stem cells, plasticity, preclinical and clinical application, medicine

\footnotetext{
${ }^{1}$ Department of Anatomy, Poznan University of Medical Sciences, Poznan, Poland

${ }^{2}$ Physiology Graduate Program, North Carolina State University, Raleigh, NC, USA

${ }^{3}$ Department of Histology and Embryology, Poznan University of Medical Sciences, Poznan, Poland

${ }^{4}$ Department of Obstetrics and Gynaecology, University Hospital and Masaryk University, Brno, Czech Republic

${ }^{5}$ Department of Biomaterials and Experimental Dentistry, Poznan University of Medical Sciences, Poznan, Poland

* Correspondence: mdyszkiewicz@ump.edu.pl

Full list of author information is available at the end of article
} 


\section{Introduction}

Arguably, the stem cell revolution began with the isolation of the first embryonic stem cell lines by Sir Martin Evans (Evans and Kaufman, 1981) with the initial application to generate functional gene knockouts, which lead to significant scientific discovery [1].

The application of stem cells is one of the subjects most studied in recent years related to treating various diseases. The possibility of implementing stem cells in Parkinson's disease, amyotrophic lateral sclerosis, spinal cord injury or stroke could revolutionize current nervous system therapies. Stem cell use in metabolic diseases, the best example is the search for their therapeutic impact on diabetes, seems an achievable application.

According to the data available on the eurostemcell website, stem cells can be divided into three basic groups:embryonic stem cells that originate from embryos, induced pluripotent stem cells, i.e. mature cells, which through the action of molecular biology become stem cells, and tissue stem cells which allow an organism to constantly regenerate selected tissues.

More specific names used for groups of stem cells are as followed: adipose-derived stem cells (ASCs), articular cartilage-resident chondroprogenitor cells (ACPCs), mesenchymal stem cells (MSCs), endometrial mesenchymal stromal cells (eMSCs), amniotic epithelial cells (AECs), cancer stem cells (CSCs), trophoblast stem cells (TSCs), neural stem cells (NSCs) and dental pulp stem cells (DPSCs).

In addition to using stem cells in clinical practice, it is equally important to understand their original properties, which should guide researchers and clinicians to their subsequent therapeutic use. Proper isolation will allow obtaining of the highest quality cells and enable efficient and safe cell culture.

\section{Origin and isolation of stem cells}

The increasing popularity of stem cells in medicine requires an improvement in cell culture methods and techniques of isolation.

\section{Adipose-derived stem cells}

Human adipose-derived stem cells (ASCs) are one of the most frequently used stem cells. ASCs have many applications because they can differentiate towards osteogenic, chondrogenic and adipogenic phenotypes [2]. The ASCs are collected via surgical excision of the adipose tissue or liposuction, which allows the collection of a large number of viable ASCs with a high proliferation rate. Also, liposuction is a safe procedure for the patient and is less invasive than surgical collection. Despite many new and experimental attempts to isolate cells, the Zuk protocol remains the most popular method [3]. One of the novelties in isolation method is the method of removing erythrocytes from the aspirate using $\mathrm{NH} 4 \mathrm{Cl}$. During some of the recent tests, a hypotonic $\mathrm{NaCl}$ solution proved to be more effective in purify- ing ASC from erythrocytes and meeting all accepted standards [4]. Enzymatic isolation methods have been more effective in acquiring proper amount of cells in comparison to non-enzymatic methods, which allow for better quality cells $[2,4]$.

\section{Articular cartilage-resident chondroprogenitor cells}

ACPCs are obtained from metacarpal cartilage using a scalpel. Subsequently, under sterile conditions, they are crushed and digested with enzymes. MSCs are isolated from a centrifuged bone marrow aspirate [5]. In addition to acquiring MSC from bone marrow aspirate, a rich source of MSCs in adults is located in fatty tissue and teeth pulp [6].

\section{Endometrial mesenchymal stromal cells}

A relatively new and poorly researched source of adult HLA-compatible stem cells (SCs) is menstrual blood, which abounds in a massive amount of endometrial mesenchymal stromal cells (eMSCs). The collection of menstrual blood can be conducted over the whole menstruation period. The collection itself lasts from 3 to 5 hours. The obtained material must be sterilized and stored under sterile conditions. The blood separator is centrifuged for approximately 5 minutes (1500 rpm). The resulting sediment is diluted, with a buffer solution consisting of $\mathrm{NH} 4 \mathrm{Cl}, \mathrm{KHCO} 3$, ethylenediaminetetraacetic acid and distilled water. The cells are then filtered and centrifuged again to form a pale pink precipitate, which can be inoculated into culture [7].

\section{Mesenchymal stem cells}

Mesenchymal stem cells (MSC) were once isolated mainly from the bone marrow. Currently, they are also obtained from adipose tissue and neonatal tissues such as the umbilical cord, placenta or amniotic fluid. The MSC method of isolation involves scraping bone fragments into heparin solution or tissue aspiration. The isolation of MSCs derived from the bone marrow is quite simple because it uses the adhesion of these cells to the plastics. Thanks to this, other cells can be separated by rinsing them out several times [8]. MSC can also be obtained from Wharton jelly after thorough cleansing from the blood. The procedure itself involves cutting the umbilical cord into small pieces and purifying it through enzymatic hydrolysis. The resulting solution is centrifuged at $300 \mathrm{~g}$ for 5 minutes and the pellet can be used for cell culture. The whole procedure must be sterile to avoid the possibility of infection $[8,9]$.

\section{Amniotic epithelial cells}

The isolation of these cells allows obtaining of cells that are morphologically similar to human fibroblasts. They are collected in two stages. First, amniotic epithelial cells are isolated, according to the Miki and Storm protocol. The AECs are then 
isolated with the participation of enzymes. It is important during isolation to avoid contaminating of the sample with hematopoietic stem cells. The procedure can be easily checked to determine whether we have carried out the entire process correctly, due to the absence of the CD34 marker AECs [10].

\section{Cancer stem cells}

Isolation is the removal of differentiated and non-malignant cells from the material taken from a digested tumour. An appropriate medium allows for a targeted slow increase in Cancer Stem Cells (CSC). Cell markers play an important role in the isolation of specific cells. Some of these markers are very similar to other stem cells. CD44 and CD133 are the most important markers that were detected the vast majority of CSCs. There are also markers characteristic of the tissue from which CSCs originate. For example, it is an increase in the epithelial cell adhesion molecule (EPCAM) in cells responsible for stomach cancer [11]. In the case of colon cancer, the marker that allows probable detection of CSCs in the tumour cell population is CD-24, CD-29 and ALDH1 [12].

\section{Trophoblast stem cells}

Trophoblast stem cells (TSCs) are isolated from the placenta during the first trimester of pregnancy. Attempts to isolate TSCs from blastocysts has proven to be ineffective. TSCs have been isolated in 3 ways. The first attempted method is based on the Percoll density gradient centrifugation. However, cells that were selected as TSCs based on surface markers accounted for about $1 \%$ of the population [13]. The second attempted method has selected a similar number of cells, but is based on flow cytometry that isolates a side population of TSCs due to faster pumping of Hoechst 33342 dye from the cytoplasm. The third method is also based on Hoechst staining following trypsin digestions [13]. Only $0.14 \%$ were isolated in Takao's work [14]. Further research is being carried out on the ability to differentiate TSCs [13].

\section{Neural stem cells}

The last group of cells mentioned in the article is Neural stem cells (NSCs). Isolated mouse NSCs originate from the thoracic segment of the spinal cord, the striatum, or the subependymal region. In adult mammals, NSC are abundantly present in the olfactory bulb. The NSC isolation itself requires epidermal growth factor and basic fibroblast growth factor. The cells can be stored in enzyme-based solution and applied repeatedly due to lack of adherent properties [15].

\section{Plasticity and differentiation of stem cells}

The proliferative index and differentiation capability of stem cells is quite attractive as a therapeutic platform. The direction of cell differentiation determines their use in research or clinical situations.

\section{Mesenchymal stem cells}

MSCs are cells with a huge potential for differentiation. It is worth mentioning that until now the most achievable directions for the differentiation of MSCs were adipocytes, chondrocytes, and osteoblasts. The MSCs' mesodermal origin is not a limitation for differentiation of their population into lines from other germ layers. Appropriate preparation of transcription factors, cytokines and media allow the production of endothelial cells, hepatocytes, epithelial cells and changes to Inducible Pluripotent Stem Cells from MSCs. Numerous MSCs have also been found around the blood vessels. Most MSCs are involved in the repair of vessels, which would confirm the possibility of differentiation of these cells into endothelial cells. Products synthesized by MSCs also affect the proper functioning of T cells. It is also indicated the important role of exosomes in the processes of these cells [6].

\section{Amnotic epithelial stem cells}

Amniotic Epithelial Stem Cells are also a group of cells with a wide range of differentiation. They can differ in the direction of all three germ layers. In animal studies, these cells were specialized in osteoblastic and chondroblastic lines. Among people, however, it was possible to obtain differentiation into cartilage [10].

\section{Adipose-derived stem cells}

ASCs are cells that have many similar properties to MSCs, due to the high immunomodulatory and secretory effect and the ability to differentiate into many cells: cardiomyocytes, muscle precursor cells, osteoblasts, chondrocytes, hepatocytes, pancreatic cells, endothelial cells, and hematopoietic-supporting. ASCs are also very plastic in response to environmental stimuli, they can survive very unfavourable conditions, to stimulate and start the repair process. Studies show that the inflammatory process significantly affects the differentiation of ASCs. The aging of the body, as well as the concentration of TNF-alpha, has a fairly large impact on the population of these cells. Obesity is a chronic inflammatory process that promotes the differentiation of ASCs into adipocytes. It is caused by both adipocyte hypertrophy and hyperplasia of ASCs. The presence of metabolic syndrome, diabetes or obesity affects the plasticity of ASCs. In these people ASCs show a higher metabolic rate than in healthy people, and the demand for ATP is similar to cancer cells. An important role during attempts to use ASCs in proangiogenic activities are individual conditioning factors and Notch signalling, which are disturbed in people with the above-mentioned disorders [16].

\section{Dental pulp cells}

Dental pulp cells (DPSCs) are similar to both embryonic stem cells and mesenchymal stem cells, and 
they can differentiate into osteoblastic, chondroblastic lines, adipogenesis, neurogenesis, odontogenesis, dentinogenesis and myogenic lines. DPSCs are often used for in-vitro culturing due to traits that facilitate differentiation into nerve tissue cells, neurosphere or directly into motor neurons. It is also possible to differentiate them into immature Schwann cells or oligodendrocytes suggesting that the success of these activities was proven by a successful attempt in animal models. DPSCs present immunomodulatory and angiogenic properties [17].

\section{Cancer stem cells}

In animal models, it was confirmed that tumour invasion cells originate from a cancer stem cell that phenotypically differs from invasive cells. In the gastrointestinal tract, it was confirmed that CSCs have the same cell markers as cells responsible for the regeneration of these organs after injury [18]. In the lungs, mutations in different cells were able to induce benign lesions, whereas only mutations in alveolar type II cells caused malignant neoplasms [19], which is similar to neural tissue tumours in mice. Only mutations in progenitor cells were able to induce malignant changes. In sarcomas, the appearance of a mutation in the stem cells may give rise to the formation of cells with a full tumour phenotype [20]. Interestingly, microenvironment has a huge impact on the maintenance of cancer cells. For example, during acute lymphoblastic leukaemia, cancer cells can protect mutated stem cells, which result in the ineffectiveness of chemotherapy in eradication of the disease. In other myeloproliferative tumours, malignant cells block normal stem cells, and CSC stimulates severe and abnormal osteogenesis, which causes even greater changes in the microenvironment indicating that the potential for CSC differentiation depends on external conditions [18].

\section{Neural stem cells}

NSCs can be obtained from both embryonic stem cells and iPSC. Differentiation can take place in Monolayer culture or embryoid body (EB) formation [15]. Somatic cells may also be transdifferentiated into NSCs (NSC Induced cells), which were successfully generated not only from mice but also from human fibroblasts [21]. Transdifferentiation was also successful with the participation of specific chemical compounds. Neuronal stem cells are obtained from fat MSCs by activating SOX1 [15].

\section{Pre-clinical application of stem cells}

Dental Pulp Stem Cells(DPSC) may play an important role in the treating diseases of the nervous system. In spinal cord injury (SRI) the role of stem cells is not only limited to the ability to differentiate and support axonal regeneration, but also to inhibit the apoptosis of spinal cord cells as a result of reducing inflammation in the damaged site.
The use of DPSC in animal models showed their angiogenic properties, which accelerated the recovery, limited the number of free radicals and, through its action to reduce inflammation, supported brain regeneration $[17,22]$.

Positive effects have also been reported in Parkinson's disease. DPSCs can be induced in vitro into dopamine-producing neurons, while in a mouse model, they restored the function of dopaminergic neurons and reduced DNA damage limiting the reactive oxygen species concentration $[17,23]$.

DPSC's protective role was observed treating Alzheimer's disease, allowing for gradual repair and stabilization of neuron cytoskeleton [17].

Neural Precursor Cells (NPC) were used to treat multiple sclerosis. In animal models, NPC transplantation improved mice remyelination progression, which resulted from their beneficial neurotrophic effects on host cells and stimulated them to accelerate remyelination. Another trial used bone marrow stem cells, which became the brain-derived neurotrophic factor (BDNF) carrier. Because NPC were directly transplanted into the CNS, the blood brain barrier was not an obstacle to BDNF allowing for delayed development of disease symptoms, less demyelination and faster remyelination in the animal model in experimental autoimmune encephalomyelitis [15].

Stem cells are also used in the treatment of eye diseases. Studies on Age-related Macular Degeneration (AMD) have shown that in mouse models, the use of human neural progenitor cells has given positive results because vision loss has been inhibited. Currently, neural progenitor cells transplants are being studied in people with AMD [15]. Currently, research on the use of human embryonic stem cells in this therapy has been conducted in nine patients. Negative effects of transplanted cells have not been demonstrated, while $72 \%$ have seen increased pigmentation at the graft site. In contrast, human induced pluripotent stem cells, exhibited no side effects, but the patient's visual acuity did not change. However, during studies with adult stem cells, three patients lost vision caused by retinal detachment [24].

Effective attempts to regenerate peripheral nerve following injuries have also been studied using DPCS in combination with biomaterials. DPCS have been able to stimulate the regeneration of facial nerve fragments or repair a damaged sciatic nerve in vitro. The combination of collagen structures together with DPSCs, which were functionally similar to Schwann cells or oligodendrocytes in laboratory experiments, significantly assisted in the repair and regeneration of damaged nerves, which was associated, among others, with the secretion of neurotrophic factors. Therefore, it is possible for their future use in situations where current methods do not guarantee the effectiveness of treatment [17].

The main goal of treating a myocardial infarction by using stem cells is to improve circulation and 
cardiogenesis at the affected site of the myocardial. Studies in animal models have been very promising and have shown tremendous therapeutic success. Particular attention should be paid to mesenchymal stemcells. In rat models, they inhibited inflammation and cardiomyocyte hypertrophy and allowed the regeneration of approximately $80 \%$ of the damaged heart fragment. In addition, they normalized the work of the whole heart. Over 3,000 people have undergone bone marrow cell transplantation. However, among the people who received similar treatment, significant improvement was not observed. One of the reasons is the different level of damage and location of each infarction [25].

Currently MyoCell, autologous stem cells derived from skeletal muscle are the most advanced in therapy in patients after myocardial infarction. The cells are injected directly into the scar tissue of the heart. In the animal testing phase, an improvement in left ventricular parameters was noticed like left ventricular ejection fraction. The next phases of research, which were already in humans, also brought positive results. There were no side effects except for possible postoperative arrhythmia episodes. Clinical trials are being continued [26].

\section{Clinical application}

MSCs from the umbilical cord or bone marrow were used in clinical trials in patients with systemic lupus erythematosus refractory to standard treatment. Cycloxoxamide was used to suppress the defensive response prior to transplantation. During therapy, only a small proportion of patients did not have a clinical response to MSC administration. The vast majority had partial or complete remission. The results in patients indicated an improvement in health even up to 4 years after starting therapy. The amount of steroids and immunosuppressants they used also decreased. Renal function improved. No side effects were reported, and the relapse rate was lower compared to previous therapies [27].

Cultured epithelial autografts are composed of keratinocytes derived from a skin fragment taken from the patient and are able to integrate and regenerate very quickly. They were used, among others, to treat people with extensive burns. Confluent or pre-confluent autologous or allogeneic keratinocytes under various trade names are in the form of aerosols and treat hard to heal wounds and ulcers. Some preparations were also enriched with fibrin glue and were used in the treatment of diabetic wounds, ulcers or burns [28].

Burn patients have been treated with stem cells. The first of these experimental treatments employed bone marrow derived cells taken from the donor iliac crest. After growth for two weeks, cells were cryopreserved and then expanded for a week in vitro and the cells were applied topically. Improvement of blood supply was observed after topical adminis- tration, and four days after the therapy, the skin autograft was accepted, and level of pain was reduced. In a second study, cells were taken from a deceased donor's iliac crest. The cells were expanded for two weeks and subjected to cryopreservation. In this case, the level of pain also decreased, blood supply and granulation tissue appeared, and after 35 days a successful skin transplantation took place. After 3 years, the skin was still in good condition [29].

In diseases of corneal damage, stem cells are used in patients with a deficiency or loss of lymphatic stem cells. Clinical autografts are still used for unilateral deficiencies and allografts for bilateral, which achieve a nearly $40 \%$ effect at 3-5 years of transplant survival. A second method is also used which uses Human amniotic membrane (HAM), collagen shields, fibrin glue, denuded anterior lens capsule and temperature sensitive biopolymers. This transplanted into the affected eye guarantees $76 \%$ success with a 1-3 year transplant survival. Attempts have been made to improve the second method by removing xenobiotics or using lenses, they have achieved $63 \%$ effectiveness 2.5 years after the procedure [30].

\section{Conclusions}

Along with the increasing knowledge about stem cells, more techniques of isolation and differentiation arise, thus increasing the quality of the research. There is a high number of preclinical studies and ongoing clinical trials on numerous stem cell therapies. Some of them might be soon introduced into the practice and few of them are already confirmed to be successful and approved for next part of clinical tests and in future they could have clinical application like a MSCs in bone injuries' therapy and stem cells in amyotrophic lateral sclerosis.

Yet, some of the limitations are not yet overcome. To achieve complete safety of the therapy the scientists must first know the molecular mechanisms driving the faith of stem cells in patient's organism. However, this research cannot be fully conducted in in vitro conditions. The effort put into these numerous ongoing trials will one day result in successful therapy.

\section{Ethical approval}

The conducted research is not related to either human or animal use.

\section{Corresponding author}

Marta Dyszkiewicz-Konwińska, Department of Biomaterials and Experimental Dentistry, 70 Bukowska St., 60-812 Poznań, Poland and Department of Anatomy, Poznan University of Medical Sciences, 6 Święcickiego St., 60-781 Poznań, Poland, Tel./Fax: +48 61 8546565, e-mail:mdyszkiewicz@ump.edu.pl.

\section{Conflict of interest statement}

The authors declare they have no conflict of interest.

\section{References}

1. Evans MJ, Kaufman MH. Establishment in culture of pluripotential cells from mouse embryos. Nature. 1981;292:154-6; DOI:10.1038/292154a0. 
2. Zou J, Wang W, Neffe AT, Xu X, Li Z, Deng Z, Sun X, Ma N, Lendlein A. Adipogenic differentiation of human adipose derived mesenchymal stem cells in 3D architectured gelatin based hydrogels (ArcGel). Clin Hemorheol Microcirc. 2017;67:297-307; DOI:10.3233/CH-179210

3. Zuk PA, Zhu M, Mizuno H, Huang J, Futrell JW, Katz AJ, Benhaim P, Lorenz HP, Hedrick MH. Multilineage Cells from Human Adipose Tissue: Implications for Cell-Based Therapies. TISSUE Eng. 2001;7.

4. Palumbo P, Lombardi F, Siragusa G, Cifone M, Cinque B, Giuliani M. Methods of Isolation, Characterization and Expansion of Human Adipose-Derived Stem Cells (ASCs): An Overview. Int J Mol Sci. 2018;19:1897; DOI:10.3390/ijms19071897.

5. Levato R, Webb WR, Otto IA, Mensinga A, Zhang Y, van Rijen M, van Weeren R, Khan IM, Malda J. The bio in the ink: cartilage regeneration with bioprintable hydrogels and articular cartilage-derived progenitor cells. Acta Biomater. 2017;61:41-53; DOI:10.1016/j.actbio.2017.08.005.

6. Guasti L, New SE, Hadjidemetriou I, Palmiero M, Ferretti P. Plasticity of human adipose-derived stem cells - relevance to tissue repair. Int J Dev Biol. 2018;62:431-9; DOI:10.1387/ijdb.180074pf.

7. Kovina M V., Krasheninnikov ME, Dyuzheva TG, Danilevsky MI, Klabukov ID, Balyasin M V., Chivilgina OK, Lyundup A V. Human endometrial stem cells: High-yield isolation and characterization. Cytotherapy. 2018;20:361-74; DOI:10.1016/j.jcyt.2017.12.012

8. Hoffmann A, Floerkemeier T, Melzer C, Hass R. Comparison of in vitro -cultivation of human mesenchymal stroma/stem cells derived from bone marrow and umbilical cord. J Tissue Eng Regen Med. 2017;11:2565-81; DOI:10.1002/term.2153.

9. Mushahary D, Spittler A, Kasper C, Weber V, Charwat V. Isolation, cultivation, and characterization of human mesenchymal stem cells. Cytom Part A. 2018;93:19-31; DOI:10.1002/cyto.a.23242.

10. Aurelio Muttini, DVM,* Barbara Barboni, DVM, PhD *, Luca Valbonetti, DVM,* Valentina Russo, DVM *, and Nicola Maffulli, MD, PhD F. Amniotic Epithelial Stem Cells: Salient Features and Possible

11. Abbaszadegan MR, Bagheri V, Razavi MS, Momtazi AA, Sahebkar A, Gholamin M. Isolation, identification, and characterization of cancer stem cells: A review. J Cell Physiol. 2017;232:2008-18; DOI:10.1002/ jcp.25759.

12. Wahab SMR, Islam F, Gopalan V, Lam AK. The Identifications and Clinical Implications of Cancer Stem Cells in Colorectal Cancer. Clin Colorectal Cancer. 2017;16:93-102; DOI:10.1016/j.clcc.2017.01.011

13. Gamage TK, Chamley LW, James JL. Stem cell insights into human trophoblast lineage differentiation. Hum Reprod Update. 2016;23:77-103 DOI:10.1093/humupd/dmw026.

14. Takao T, Asanoma K, Kato K, Fukushima K, Tsunematsu R, Hirakawa T, Matsumura S, Seki H, Takeda S, Wake N. Isolation and Characterization of Human Trophoblast Side-Population (SP) Cells in Primary Villous Cytotrophoblasts and HTR-8/SVneo Cell Line. PLoS One. 2011;6:e21990 DOI:10.1371/journal.pone.0021990.

15. Tang Y, Yu P, Cheng L. Current progress in the derivation and therapeutic application of neural stem cells. Cell Death Dis. 2017;8:e3108-e3108 DOI:10.1038/cddis.2017.504.

16. Badimon L, Cubedo J. Adipose tissue depots and inflammation: effects on plasticity and resident mesenchymal stem cell function. Cardiovasc Res. 2017;113:1064-73; DOI:10.1093/cvr/cvx096.

17. Luo L, He Y, Wang X, Key B, Lee BH, Li H, Ye Q. Potential Roles of Dental Pulp Stem Cells in Neural Regeneration and Repair. Stem Cells Int 2018;2018:1-15; DOI:10.1155/2018/1731289.

18. Campos-Sánchez E, Cobaleda C. Tumoral reprogramming: Plasticity takes a walk on the wild side. Biochim Biophys Acta - Gene Regul Mech 2015;1849:436-47; DOI:10.1016/j.bbagrm.2014.07.003.

19. Zhu L, Gibson P, Currle DS, Tong Y, Richardson RJ, Bayazitov IT, Poppleton H, Zakharenko S, Ellison DW, Gilbertson RJ. Prominin 1 marks intestinal stem cells that are susceptible to neoplastic transformation. Nature. 2009;457:603-7; DOI:10.1038/nature07589.

20. Straessler KM, Jones KB, Hu H, Jin H, van de Rijn M, Capecchi MR. Mod eling Clear Cell Sarcomagenesis in the Mouse: Cell of Origin Differentiation State Impacts Tumor Characteristics. Cancer Cell. 2013;23:215-27 DOI:10.1016/J.CCR.2012.12.019

21. Shahbazi E, Moradi S, Nemati S, Satarian L, Basiri M, Gourabi H, Zare Mehrjardi N, Günther P, Lampert A, Händler K, Hatay FF, Schmidt D, Molcanyi M, Hescheler J, Schultze JL, Saric T, Baharvand H. Conversion of Human Fibroblasts to Stably Self-Renewing Neural Stem Cells with Single Zinc-Finger Transcription Factor. Stem Cell Reports. 2016;6:53951; DOI:10.1016/J.STEMCR.2016.02.013.

22. Leong WK, Lewis MD, Koblar SA. Concise Review: Preclinical Studies on Human Cell-Based Therapy in Rodent Ischemic Stroke Models: Where Are We Now after a Decade? Stem Cells. 2013;31:1040-3; DOI:10.1002/ stem.1348.
23. Wang Y, Chen S, Yang D, Le W. Stem Cell Transplantation: A Prom ising Therapy for Parkinson's Disease. J Neuroimmune Pharmacol. 2007;2:243-50; DOI:10.1007/s11481-007-9074-2.

24. Higuchi A, Kumar SS, Benelli G, Alarfaj AA, Munusamy MA, Umezawa A Murugan K. Stem Cell Therapies for Reversing Vision Loss. Trends Biotechnol. 2017;35:1102-17; DOI:10.1016/j.tibtech.2017.06.016.

25. Michler RE. The current status of stem cell therapy in ischemic heart disease. J Card Surg. 2018;33:520-31; DOI:10.1111/jocs.13789.

26. Haider HK, Lei Y, Ashraf M. MyoCell, a cell-based, autologous skeleta myoblast therapy for the treatment of cardiovascular diseases. Curr Opin Mol Ther. 2008;10:611-21.

27. Wang D, Zhang H, Liang J, Li X, Feng X, Wang H, Hua B, Liu B, Lu L, Gilkeson GS, Silver RM, Chen W, Shi S, Sun L. Allogeneic Mesenchymal Stem Cell Transplantation in Severe and Refractory Systemic Lupus Erythematosus: 4 Years of Experience. Cell Transplant. 2013;22:2267-77; DOI:10.3727/096368911X582769c.

28. Ojeh N, Pastar I, Tomic-Canic M, Stojadinovic O. Stem Cells in Skin Regeneration, Wound Healing, and Their Clinical Applications. Int J Mol Sci. 2015;16:25476-501; DOI:10.3390/ijms161025476.

29. Ahmadi AR, Chicco M, Huang J, Qi L, Burdick J, Williams GM, Cameron AM, Sun Z. Stem cells in burn wound healing: A systematic review of the literature. Burns. 2019;45:1014-23; DOI:10.1016/j.burns.2018.10.017.

30. Ljubimov A V, Saghizadeh M. Progress in corneal wound healing. Prog Retin Eye Res. 2015;49:17-45; DOI:10.1016/j.preteyeres.2015.07.002. 